\section{Central Nervous System Vasculitis: All That Glitters Is Not Gold}

To the Editor:

Primary angiitis of the central nervous system (PACNS) is a vasculitis affecting small to medium-size arteries of any part of the brain, spinal cord, and meninges, resulting in highly variable and nonspecific manifestations. This can pose a significant diagnostic challenge because the clinical and radiological features of PACNS can mimic a number of other disorders. We describe a patient who presented with clinical and radiological manifestations compatible with PACNS but in whom brain biopsy disclosed a strikingly different diagnosis.

A 50-year-old woman presented in January 2009 with a 2-month history of impaired gait associated with bilateral leg weakness, which progressed rapidly over the 10 days prior to her admission to the extent that she was unable to walk independently. Examination revealed positive upper motor neuron signs in the lower legs and some positive cerebellar signs. Laboratory examination revealed normocytic normochromic anemia (hemoglobin $9.7 \mathrm{~g} / \mathrm{dl}$; normal 12-16 g/dl) and markedly raised inflammatory markers [C-reactive protein (CRP) $92 \mathrm{mg} / \mathrm{l}$; normal $<5 \mathrm{mg} / \mathrm{l}$; erythrocyte sedimentation rate (ESR) $110 \mathrm{~mm} / \mathrm{h}$; normal $<10 \mathrm{~mm} / \mathrm{h}$ ]. The rest of her blood laboratory examination was unremarkable. A working diagnosis was made of cerebrovascular accident.

The patient had been feeling unwell for the previous 2 years with unexplained anemia and persistently raised inflammatory markers (ESR, CRP). Despite multiple investigations [including bone marrow examination and computed tomography (CT) scans of thorax and abdomen], her symptoms, anemia, and the raised inflammatory markers remained unexplained. During this period she required periodic red blood cell transfusions, but she received no other therapy.

Within 24 hours of her hospital admission, her clinical condition deteriorated further. Her Glasgow coma scale (GCS) dropped to 6/15, and magnetic resonance imaging (MRI) of the brain showed diffuse areas of increased $\mathrm{T} 2$ signals in the cortical gray matter and overlying meninges bilaterally, and these were across multiple vascular territories (Figure 1). Intracranial MR angiogram and CT angiogram of intracranial arteries were unremarkable. Diffusion-weighted images and MRI of the brain with gadolinium was performed (Figure 1). Lumbar puncture fluid examination revealed white cell count 4 with lymphocyte predominance, no microorganisms, glucose $4.7 \mathrm{mmol} / \mathrm{l}$, total protein $0.88 \mathrm{~g} / \mathrm{l}$. Further examination revealed the following to be normal/negative: CT thorax, abdomen and pelvis, repeated blood cultures, transesophageal echocardiogram, antinuclear antibody, extractable nuclear antigen, and antineutrophil cytoplasmic autoantibodies.

Empiric treatment with methylprednisolone pulses $1 \mathrm{~g}$ daily for 3 days was administered. An immediate therapeutic response was noted with improvement in GCS to 14/15 in the next 3 days, and improvement in cognitive function. A working diagnosis of cerebral vasculitis was made on the basis of compatible clinical history, elevated inflammatory markers, anemia of chronic disease, and typical MR features.

Brain and meningeal biopsy showed diffuse abnormally large B cell infiltration of cerebral vessels typical of intravascular lymphoma (IVL; Figure 2). The bone marrow examination again was normal. She subsequently had 8 cycles of chemotherapy involving rituximab, cyclophosphamide, doxorubicin, vincristine, and prednisolone, and she also underwent stem cell transplantation. Her corticosteroids were gradually reduced. Currently, her lymphoma is in complete remission with rituximab infusions every 3 months, and a recent positron emission tomography scan was consistent with this clinical remission.

IVL is a rare subset of extranodal diffuse lymphoma. It is characterized by the luminal proliferation of lymphoid cells within capillaries and medium-size vessels, and there is little or no involvement of the parenchyma of the concerned organ. IVL can mimic both clinical and radiological features of PACNS; however, radiologic findings are nonspecific for both of these diseases. Constitutional symptoms such as fever, malaise, weight loss, and arthralgias are common in IVL but less common in PACNS; acute-phase reactants are typically markedly elevated in IVL, but these tend to be normal in PACNS; and lumbar puncture fluid is usually abnormal in PACNS (modest, nonspecific elevations in total protein level and a lymphocytic pleiocytosis) ${ }^{1}$. Moreover, cerebral angiography, even conventional catheter-directed angiography, is often normal in PACNS because small vessel involvement cannot be detected ${ }^{2}$.
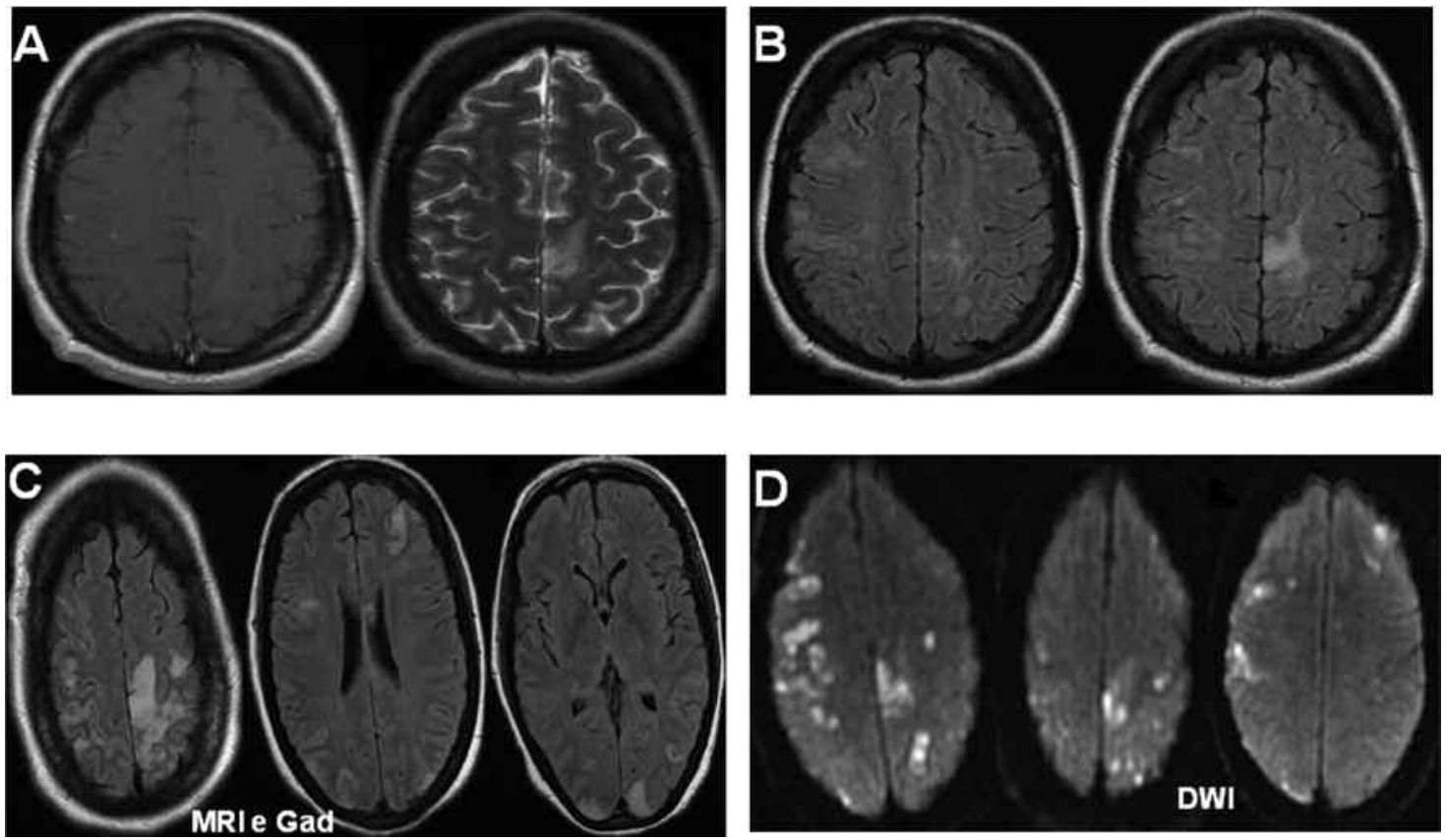

Figure 1. MRI of the brain. A. T1-weighted images showing subcortical and meningeal high signal intensity. B. T2 FLAIR images. C. MRI with gadolinium showing multifocal gray matter abnormalities. D. Diffusion-weighted images confirmed multiple gray matter infarctions.

Personal non-commercial use only. The Journal of Rheumatology Copyright @ 2012 . All rights reserved. 

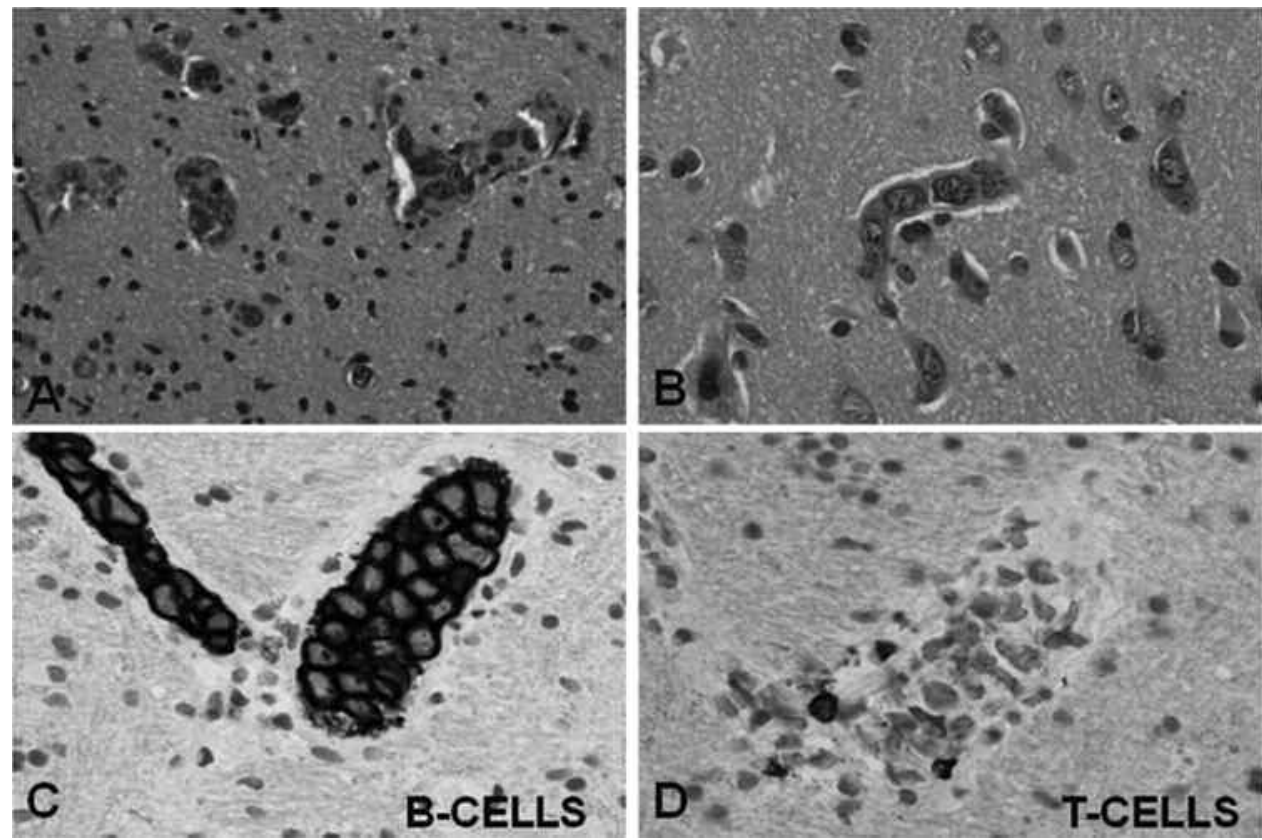

Figure 2. A and B. H\&E staining showing all intracortical vessels stuffed with abnormally large cells. C and D. Immunophenotyping reveals the tumor cells as large B cells.

Clinicians are frequently reluctant to request a brain biopsy given the invasive nature of this procedure. However, given the inability to confirm the diagnosis of PACNS on the basis of clinical data, radiologic studies, and cerebral angiography, and the significant potential toxicity of immunosuppressive regimens used for PACNS, every effort should be made to establish diagnostic certainty by obtaining tissue biopsy. Moreover, CNS biopsy can be an invaluable tool to rule out mimics of PACNS, because alternative diagnoses can be made in $39 \%$ of cases of suspected PACNS ${ }^{3}$. The sensitivity of brain biopsy for diagnosing PACNS is about $75 \%-80 \%$, and in most reports brain biopsy has had a morbidity of about $2 \%$, with a negligible mortality when done by an experienced neurosurgeon ${ }^{4}$. Our case highlights the critical role of tissue biopsy in the evaluation of patients with suspected PACNS to help establish the correct diagnosis and to implement appropriate treatment.

MUHAMMAD HAROON, MB, MMedSc, MRCPI; EAMONN MOLLOY, MD, MS, MRCPI, Department of Rheumatology, Bone and Joint Unit, St. Vincent's University Hospital, Dublin; MICHAEL FARRELL, FRCPI, FRCPC, FRCPath, Professor, Department of Neuropathology, Beaumont Hospital, Dublin; SHAFEEQ ALRAQI, MRCP (UK), Department of Rheumatology, Bone and Joint Unit, St. Vincent's University Hospital, Dublin, Ireland. Address correspondence to Dr. M. Haroon, Department of Rheumatology, Bone and Joint Unit, St. Vincent's University Hospital, Dublin, Ireland. E-mail: mharoon301@ hotmail.com

\section{REFERENCES}

1. Calabrese LH, Duna GF, Lie JT. Vasculitis in the central nervous system. Arthritis Rheum 1997;40:1189-201.

2. Benseler SM. Central nervous system vasculitis in children. Curr Rheumatol Rep 2006;8:442-9.

3. Alrawi A, Trobe JD, Blaivas M, Musch DC. Brain biopsy in primary angiitis of the central nervous system. Neurology 1999;53:858-60.

4. Parisi JE, Moore PM. The role of biopsy in vasculitis of the central nervous system. Semin Neurol 1994;14:341-8.

J Rheumatol 2012;39:3; doi:10.3899/jrheum.111141 